Annuaire suisse de politique de développement

27-2 | 2008

Migration et développement: un mariage arrangé

\title{
Migration et développement : une histoire de brouilles et de retrouvailles
}

\section{Alessandro Monsutti}

\section{(2) OpenEdition}

1 Journals

Édition électronique

URL : http://journals.openedition.org/aspd/162

DOI : 10.4000/aspd.162

ISSN : 1663-9669

Éditeur

Institut de hautes études internationales et du développement

Édition imprimée

Date de publication : 1 décembre 2008

Pagination : 23-42

ISBN : 978-2-940415-07-6

ISSN : 1660-5934

Référence électronique

Alessandro Monsutti, «Migration et développement : une histoire de brouilles et de retrouvailles », Annuaire suisse de politique de développement [En ligne], 27-2 | 2008, mis en ligne le 01 avril 2010, consulté le 08 septembre 2020. URL : http://journals.openedition.org/aspd/162 ; DOI : https://doi.org/ 10.4000/aspd.162 


\title{
Migration et développement: une histoire de brouilles et de retrouvailles
}

\author{
Alessandro Monsutti*
}

\section{Migration et développement: I'optimisme en perspective}

Bien qu'elle eût suscité dès l'après-guerre des débats passionnés, la question de la relation entre migration et développement (migration-development nexus en anglais ${ }^{1}$ ) a acquis une visibilité importante depuis quelques années aux niveaux politique et académique. Celle-ci est liée à l'intérêt croissant qui entoure les transferts de fonds (remittances) des migrants comme instrument pour lutter contre la pauvreté et alimenter la croissance économique ${ }^{2}$. Après les attentats du 11 septembre 2001, la discussion touche également des questions de sécurité internationale, incluant notamment le combat contre le terrorisme ou le blanchiment d'argent sale, ainsi que le rôle des diasporas dans les conflits et dans la construction de la paix.

Les organismes internationaux et les autorités étatiques prêtent ainsi une attention croissante aux migrations, comme en témoigne la création à Genève en 2003 de la Commission mondiale sur les migrations internationales (Global Commission on International Migration, GCIM). Celle-ci reçut le mandat de proposer un cadre pour formuler une réponse globale et cohérente au problème des migrations. Dans son rapport final ${ }^{3}$, la commission émet un certain nombre de recommandations pour coordonner la gestion des flux migratoires aux niveaux national et international. Le texte souligne que le nombre de migrants va continuer à croître dans les prochaines années pour des raisons structurelles (écarts démographiques, disparités économiques et politiques, etc.). Ce constat devrait être reconnu par les Etats qui sont dès lors encouragés à mettre sur pied une politique pragmatique. Il s'agirait de reconnaître l'apport des migrants à l'économie et à la vie sociale des pays d'accueil, et de tirer parti de ces mouvements humains pour promouvoir un développement plus équitable, lutter contre la pauvreté ou encore favoriser la démocratisation de régimes autoritaires. En

* Chargé de cours à l'Institut de hautes études internationales et du développement, Genève.

Mes plus vifs remerciements vont à Dawn Chatty et à Gilbert Rist pour leurs précieux commentaires, ainsi qu'à Denise Efionayi-Mäder, Jean-Claude Huot, Gérard Perroulaz et Catherine Schümperli Younossian.

1 Voir le numéro spécial de International Migration, vol. 40, nº 5, 2002, traitant de la relation entre migration et développement; en particulier, les textes introductifs de: N. Nyberg-Sørensen, N. Van Hear and P. Engberg-Pedersen, «The Migration-Development Nexus : Evidence and Policy Options State-of-the-art Overview », pp. 3-47, et «The Migration-Development Nexus : Evidence and Policy Options », pp. 49-73.

2 D. Kapur, Remittances: The New Development Mantra?, G-24 Discussion Paper Series, $\mathrm{n}^{\circ} 29$, New York; Geneva, United Nations Conference on Trade and Development, 2004.

3 Migration in an Interconnected World: New Directions for Action, Geneva, Global Commission on International Migration, October 2005. 
automne 2006, la 61 session des Nations unies s'est d'ailleurs ouverte avec le Dialogue de haut niveau sur les migrations internationales et le développement. Il s'agit de la toute première manifestation de ce genre. Cela prouve la place que la volonté de comprendre et de gérer de façon globale les migrations a acquise dans l'agenda de la communauté internationale.

Si la tentation sécuritaire est aujourd'hui marquée, elle s'accompagne paradoxalement d'un optimisme parfois un peu béat sur la contribution économique, politique et sociale des migrants à leurs régions d'origine. Cette appréciation positive des effets des migrations sur le développement du Sud est nourrie par toutes sortes de recherches mandatées et s'est imposée dans les discours politiques. Elle prévaut aujourd'hui à la Banque mondiale ${ }^{4}$, à l'Organisation internationale pour les migrations ${ }^{5}$, mais aussi à la Direction du développement et de la coopération (DDC) et dans d'autres agences nationales de développement ${ }^{6}$, qui mettent toutes la problématique des migrations au cœur de leur réflexion. Avec une frappante convergence de vues, ces institutions se proposent par leurs activités de réduire les risques que les flux migratoires occasionnent pour les personnes en mouvement comme pour les Etats, tout en soutenant les effets positifs des migrations internationales sur le développement des pays du Sud. Une situation win-win, où les intérêts des migrants originaires du Sud et ceux des Etats du Nord coïncideraient, semble être considérée comme possible dans ce discours institutionnel.

Cette rhétorique s'appuie sur les énormes montants que les migrants envoient dans leurs pays d'origine. Largement supérieurs à l'aide publique au développement (APD) ${ }^{7}$, ils sont en outre censés avoir augmenté au cours de ces dernières années (voir tableau 1$)^{8}$.

4 Voir S.M. Maimbo and D. Ratha (eds.), Remittances: Development Impact and Future Prospects, Washington, DC, World Bank, 2005 (ce volume collectif reprend, entre autres, une étude très souvent citée: D. Ratha, «Workers' Remittances: An Important and Stable Source of External Development Finance», Global Development Finance 2003, Washington, DC, World Bank, 2003, pp. 157-175). Pour l'Asie, voir Converting Migration Drains into Gains: Harnessing the Resources of Overseas Professionals, Manila, Asian Development Bank, 2006.

5 Cette dernière participe très activement à ce mouvement. Parmi une foison de publications, mentionnons par exemple Migration for Development: Within and Beyond Frontiers, Geneva, International Organization for Migration, 2006. Cet ouvrage est présenté comme une contribution au « global effort to create measures that will maximize the potential development benefits of migration and mitigate its negative aspects» (<http://www.iom.int/jahia/Jahia/cache/offonce/pid/1674?entryId=10851>). Voir également N. Baruah, Remittances to Least Developed Countries (LDCs): Issues, Policies, Practices and Enhancing Development Impact, Geneva, International Organization for Migration, 2006; et B. Gosh, Migrants' Remittances and Development: Myth, Rhetoric and Realities, Geneva, International Organization for Migration; The Hague, The Hague Process on Refugees and Migration, 2006.

6 Voir par exemple les sites Internet des agences de développement étasunienne, britannique, allemande ou suisse: United States Agency for International Development (USAID), <http://www.usaid.gov>; Department for International Development (DFID), <http://www.dfid.gov.uk/migration.asp>; Gesellschaft für Technische Zusammenarbeit (GTZ), <http://www.gtz.de/de/themen/wirtschaft-beschaeftigung/15634 htm>; Direction du développement et de la coopération (DDC), <http://www.deza.admin.ch/fr/Accueil/ Themes/Migrations>. Le DFID a plus particulièrement soutenu la création d'une plate-forme pour informer les migrants sur les meilleures possibilités de transférer de l'argent vers leurs pays d'origine, $<$ http://www.sendmoneyhome.org $>$.

7 Alors qu'ils ne se monteraient qu'à la moitié des transferts de fonds informels, les envois d'argent que les migrants effectuent par des canaux officiels ont pu représenter à eux seuls jusqu'au triple de l'aide publique au développement (Migration in an Interconnected World, op. cit., pp. 26 et 85).

8 Les transferts effectués par les migrants par des canaux officiels ont ainsi plus que doublé entre 2001 et 2006. Il faut toutefois souligner que cette augmentation reflète également le contrôle accru que les Etats exercent sur les remittances après les attentats du 11 septembre 2001. 
Tableau 1 : Evolution des transferts de fonds des migrants en comparaison avec l'aide publique au développement, 2000-2006 (en milliards de dollars)

\begin{tabular}{lrrrrrrr}
\hline Par région & 2000 & 2001 & 2002 & 2003 & 2004 & 2005 & 2006 \\
\hline Asie de l'Est et Pacifique & 17 & 20 & 20 & 35 & 39 & 45 & 47 \\
\hline Europe et Asie centrale & 13 & 13 & 14 & 17 & 23 & 31 & 32 \\
\hline Amérique latine et Antilles & 20 & 24 & 28 & 35 & 41 & 48 & 53 \\
\hline Moyen-Orient et Afrique du Nord & 13 & 15 & 16 & 20 & 23 & 24 & 25 \\
\hline Asie du Sud & 17 & 19 & 24 & 31 & 31 & 36 & 41 \\
\hline Afrique subsaharienne & 5 & 5 & 5 & 6 & 8 & 9 & 9 \\
\hline Total transferts de fonds & 85 & 96 & 117 & 145 & 165 & 193 & 206 \\
\hline Aide publique au développement & 54 & 52 & 58 & 69 & 79 & 107 & 104 \\
\hline
\end{tabular}

Source pour les transferts de fonds: World Bank, Global Development Finance 2007, Washington DC, World Bank, 2007, p. 54. Source pour l'APD: Organization for Economic Cooperation and Development, OECD.StatExtracts, <http://stats.oecd.org/wbos/ Index.aspx?DatasetCode=0DA_DONOR $>$ (consultation: 25 juin 2008).

Cependant, cet optimisme recèle un premier paradoxe: la migration devient le principe de sa négation. En effet, selon les discours des organisations internationales et des agences bilatérales de coopération, les migrants peuvent contribuer à développer leurs pays d'origine, alors que le développement peut contribuer de façon plus générale à tarir les flux migratoires. Les discours politiques qui dominent en ce début du $\mathrm{XXI}^{\mathrm{e}}$ siècle mettent l'accent sur la diminution des migrations internationales par le développement des pays du Sud, mais aussi sur la diminution de la pauvreté et l'exclusion sociale grâce aux transferts de fonds et - de façon générale - ils privilégient jusqu'au fétichisme une vision volontariste d'un développement par le bas, non institutionnalisé. Disposant de plus de fonds que l'APD, les migrants sont présentés comme des investisseurs potentiels, comme le principal moteur du développement ${ }^{9}$. On peut se demander ainsi si cette orthodoxie quelque peu stéréotypée et redondante ne contribue pas à discréditer les interventions bilatérales et multilatérales et si elle ne tend pas à légitimer le renforcement des politiques sécuritaires. Gérer les migrations cacherait en fait la volonté de les contrôler ${ }^{10}$.

Nous sommes ici face à un deuxième paradoxe. D'une part, la théorie néolibérale, qui inspire aujourd'hui les discours dominants sur la scène internationale, attribue un rôle limité à l'Etat et valorise les initiatives qui émanent d'individus ou d'associations de la société civile. D'autre part, les recommandations de la Commission mondiale sur les migrations internationales concernant la nécessité d'une coordination interétatique accrue n'ont guère eu d'effets sur les politiques concrètes; les Etats s'attachent à ce bastion de souveraineté que représente le contrôle des flux migratoires sur leurs territoires et peinent à s'entendre pour mettre sur pied un régime global visant à gérer la question (les accords de coopération qui existent portent essentiellement sur des questions sécuritaires).

9 Voir par exemple le titre éloquent d'un rapport de la parlementaire britannique H. Harman, The Hidden Heroes of International Development: Remittances from the Southwark African Diaspora to Families and Villages in Africa, London, House of Commons, 2007.

10 Th. Faist, «Migrants as Transnational Development Agents: An Inquiry into the Newest Round of the Migration-Development Nexus », Population, Space and Place, vol. 14, 2008, pp. 21-42.

Pour une critique sans ambages des mesures sécuritaires prises au Nord comme au Sud, voir M.-C. Caloz-Tschopp, Les étrangers aux frontières de l'Europe et le spectre des camps, Paris, La Dispute, 2004 ; N. Bagayoko-Penone et B. Hours, Etats, ONG et production des normes sécuritaires dans les pays du Sud, Paris, L'Harmattan, 2006. 
Les enjeux théoriques et pratiques des discussions contemporaines ainsi que la nouvelle vague d'optimisme portée par les institutions internationales et certaines agences de développement seront mis en perspective dans ce texte et plus généralement dans ce volume. Le vaste corpus de données empiriques accumulées depuis plusieurs décennies tend à démontrer qu'aucun lien mécanique entre migration et développement ou entre migration et réduction de la pauvreté ne peut être inféré à partir des divers contextes particuliers. Avançons ainsi quelques thèses:

1. Il n'y a pas de relation inversement proportionnelle entre migration et développement: les politiques «le développement pour diminuer les migrations» ou «les migrations au service du développement» ne prennent pas la mesure de la complexité et de la diversité des situations.

2. Il faut se méfier de l'idée simpliste selon laquelle la pauvreté nourrit la migration; en général, ce ne sont pas les plus pauvres qui migrent (ce constat peut d'ailleurs être étendu, avec certaines nuances, à des situations de conflit et aux réfugiés).

3. Ce ne sont pas les pays avec les PNB les plus bas ni la croissance démographique la plus haute qui ont le taux le plus élevé de migration vers l'Occident. Parmi les principaux pays d'émigration d'aujourd'hui, on compte le Mexique, le Maroc, la Turquie ou les Philippines, autrement dit des pays qui ont un niveau moyen de richesse sur le plan mondial.

4. Au cours des dernières décennies, le nombre de migrants a augmenté plus vite en Europe (l'ancien bloc soviétique inclus), en Amérique du Nord et en Australie que dans le reste du monde. Toutefois, les migrations internationales ne peuvent être réduites à un flux massif du Sud vers le Nord. Une proportion importante de la population mondiale de migrants, dont une large majorité des réfugiés, vivent dans des pays en voie de développement.

5. L'optimisme concernant les effets du développement pour faire diminuer les migrations internationales reproduit le mythe sédentariste des communautés rurales: «Un paysan heureux ne bouge pas; il reste chez lui !»

6. Les migrations sont un phénomène constitutif de l'histoire humaine. Elles ont toutefois pris une forme particulière aujourd'hui avec les nouvelles technologies de la communication et l'amélioration des moyens de transport. Cela fournit aux migrants la possibilité d'intensifier les contacts qu'ils maintiennent entre eux et avec les non-migrants restés dans le pays d'origine. Ces flux d'informations modifient les aspirations des gens (nouvelles attentes, nouvelles possibilités, nouveaux moyens...). Comment, dans un tel contexte, empêcher les gens d'être mobiles?

7. Les discours institutionnels qui célèbrent le lien entre migration et développement et qui valorisent le rôle des migrants dans l'économie et la société de leurs régions d'origine ouvrent des possibilités d'action qui sont prometteuses, même si celles-ci ne peuvent remplacer l'APD. Ils ont toutefois de faibles répercussions sur les politiques migratoires et développementales des pays de l'Organisation de coopération et de développement économiques (OCDE). Malgré la publicité qui l'entoure, la dimension migratoire ne sort guère de la rhétorique officielle des organisations internationales et des agences nationales de coopération pour être réellement prise en compte dans les projets de déve- 
loppement ${ }^{11}$. La Commission mondiale sur les migrations internationales a certes recommandé de renforcer la gestion des flux migratoires à un niveau supraétatique tout en reconnaissant leur potentiel pour le développement des pays d'origine et leur contribution à la prospérité des pays de destination. Mais les gouvernements restent attachés à leurs prérogatives en la matière.

\section{Un regard rétrospectif: périodisation des théories}

Le discours dominant quant à l'apport positif des migrants au développement des pays du Sud reflète peut-être la prééminence du paradigme néolibéral et de l'économie néoclassique. Il dénote dans tous les cas d'une certaine amnésie. Rappelons en effet que le débat en sciences sociales sur la relation entre migration et développement se place dans une tradition qui remonte à l'après-guerre, lorsque le système des institutions internationales s'est mis en place. Il est donc nécessaire de jeter un regard rétrospectif sur les théories qui ont marqué ces soixante dernières années et qui ont traversé toutes les disciplines, de l'économie à l'anthropologie, des sciences politiques à la sociologie.

Comme le montre très bien Hein de Haas, les débats théoriques ont connu un mouvement pendulaire ${ }^{12}$. Les opinions ont oscillé entre l'optimisme lié à la théorie de la modernisation dans les années 1950 et 1960 et le scepticisme inspiré par la théorie de la dépendance dans les années 1970 et même 1980. Ces deux postures ont des implications opposées pour la question du rôle des migrants, bien que celle-ci n'ait pas toujours été abordée de façon explicite dans la controverse autour du développement. Alors que les uns considéraient que les migrations internationales allaient contribuer à une allocation optimale des ressources et constituaient une étape nécessaire au développement du tiers-monde, les autres y voyaient le reflet de relations inégales que l'expansion du capitalisme ne faisait qu'accroître. Le tournant transnational, qui s'est amorcé au cours des années 1990 dans l'étude des migrations, a conduit à une vision nuancée de la relation entre lieux d'origine et pays d'accueil en soulignant que le migrant entretenait des liens multiples et pouvait être acteur à part entière de plusieurs sociétés à la fois. Certains développements de l'économie des migrations ont montré par ailleurs que la décision de migrer était prise de façon conjointe par les migrants et les non-migrants; elle concerne donc l'ensemble de l'unité domestique, qui tend par la dispersion géographique de ses membres à diversifier les sources de revenu et à gérer les risques. En parallèle, la dimension genre des migrations internationales, qui avait longtemps été occultée par les chercheurs comme par les politiques, a acquis une visibilité accrue. Si la proportion de femmes parmi les migrants n'a pas augmenté de façon spectaculaire entre

11 A titre d'exemple, mentionnons que la dimension migratoire est peu intégrée aux projets de développement des membres de la Fédération genevoise de coopération (voir la brève analyse de Christelle Genoud et Yanik Marguerat dans ce volume). Au moment où cet ouvrage était en préparation, la Direction du développement et de la coopération n'avait par ailleurs pas encore défini de politique concrète en la matière (voir la contribution de Gérard Perroulaz dans ce volume).

12 Voir en particulier un texte auquel la présentation qui suit doit beaucoup: H. de Haas, Migration and Development: A Theoretical Perspective, Oxford, International Migration Institute, University of Oxford, 2008. Plus généralement, sur les débats entre diverses écoles développementalistes, voir G. Rist, Le développement: histoire d'une croyance occidentale, Paris, Presses de Sciences Po, 2007 [ $3^{\mathrm{e}}$ édition], en particulier les pages 153-200 pour les théories de la modernisation et de la dépendance. 
1960 et aujourd'hui1 ${ }^{13}$, la restructuration économique globale a abouti à une nouvelle division internationale du travail. Le poids économique des femmes à l'extérieur de la sphère domestique ainsi que leur rôle dans le développement des pays d'origine sont aujourd'hui largement reconnus ${ }^{14}$. De très nombreuses femmes migrent de façon autonome, poursuivant un projet de vie personnel tout en restant parfois les principales pourvoyeuses de leurs familles dans le lieu d'origine ${ }^{15}$.

Voici quelques repères chronologiques approximatifs :

ـ Années 1950 et 1960: l'optimisme développementaliste lié à la théorie de la modernisation domine; les migrations contribuent au développement des pays du tiers-monde.

• Années 1970 et 1980: le scepticisme prôné par les adeptes de la théorie de la dépendance, puis par les théoriciens du système monde et les néomarxistes, prend le contre-pied de la vision précédente; les flux migratoires sont l'expression des rapports globaux de domination.

- Années 1990: une période de doutes diffus s'installe en sciences sociales, faisant écho au mouvement postmoderne et à la critique du «grand récit» des Lumières ${ }^{16}$; elle s'accompagne de l'émergence de vues nuancées sur la relation entre migration et développement liées à la nouvelle économie des migrations pour le travail (new economics of labor migration, NELM), ainsi qu'au tournant transnational et à la prise en compte accrue de la dimension genre dans l'étude des migrations.

- Années 2000: une nouvelle vague d'optimisme portée par les institutions internationales et les agences de développement s'impose; elle s'appuie sur la valorisation du rôle des migrants dans le développement des pays du Sud par les transferts de fonds.

\section{L'optimisme de l'économie néoclassique et de la théorie de la modernisation}

La théorie de la modernisation s'est structurée dans les années 1960 autour de l'œuvre de W.W. Rostow ${ }^{17}$. Elle repose sur le modèle de l'économie néoclas-

13 F. Reysoo, «Féminisation de la migration», in F. Reysoo et Ch. Verschuur (dir.), Femmes en mouvement: genre, migrations et nouvelle division internationale du travail, Berne, Commission suisse pour l'UNESCO; Direction du développement et de la coopération; Genève, Institut universitaire d'études du développement, 2004, p. 21.

14 Voir par exemple B. Ehrenreich and A.R. Hochschild, The Global Women: Nannies, Maids and Sex Workers in the New Economy, New York, Metropolitan Books, 2003, ou P. Pessar and S.J. Mahler, «Transnational Migration: Bringing Gender In», International Migration Review, vol. 37, n 3, 2003 , pp. 812-846.

15 Ce cas de figure est particulièrement courant aux Philippines, où la proportion de femmes parmi les migrants se monte à $80 \%$; voir Ch. Verschuur, «Un regard genré sur les migrations », in F. Reysoo et Ch. Verschuur (dir.), op. cit., p. 13. Voir également la contribution de Patricia A. Sto. Tomas dans ce volume.

16 Selon la fameuse formulation de J.-F. Lyotard, La condition postmoderne, Paris, Minuit, 1979.

17 W.W. Rostow, The Stages of Economic Growth: A Non-Communist Manifesto, London; Cambridge, Cambridge University Press, 1960. Comme l'indique le sous-titre, il s'agissait aussi, par la théorie de la modernisation, de proposer un modèle de développement alternatif à celui préconisé par l'Union soviétique (les deux approches partageaient pourtant les mêmes présupposés évolutionnistes d'une histoire humaine faite de stades par lesquels toute société devait passer; voir G. Rist, op. cit., pp. 164-171). 
sique, autrement dit sur une théorie de l'équilibre qui vise à une allocation optimale des ressources. Dans un tel cadre explicatif, les migrants sont censés se déplacer des régions à faible revenu vers les régions à revenu élevé, et des régions densément peuplées vers les régions moins peuplées.

Au niveau microéconomique, les migrations sont le résultat des choix rationnels des individus, qui effectuent un calcul des coûts et des bénéfices escomptés dans l'espoir de maximiser leur intérêt. Au niveau macroéconomique, les mouvements migratoires résultent d'une différence entre l'offre et la demande sur le marché du travail. Les candidats à l'emploi vont quitter les endroits où les salaires sont bas et les bras en surnombre (les zones rurales qui connaissent une mécanisation de l'agriculture) pour se rendre dans les régions qui ont besoin d'une force de travail supplémentaire et où les rémunérations sont plus élevées (les centres urbains et les zones industrielles); autrement dit, on assiste à un mouvement de travailleurs "from labor-abundant to labor-scarce countries ${ }^{18}$. La main-d'œuvre devient ainsi progressivement moins rare dans les régions de destination des migrants et plus rare dans leurs lieux d'origine. En principe, les salaires devraient donc y augmenter.

Dans ce modèle, les capitaux et les personnes circulent en sens inverses. Les migrations nationales et internationales sont considérées comme un catalyseur de changement social; elles constituent une étape nécessaire qui permettra aux sociétés traditionnelles - qui se caractérisent par un faible niveau de productivité et un usage réduit des ressources naturelles - d'accéder aux bienfaits de la modernité. L'exode rural, par exemple, participe d'un processus de développement global qui consolide la production industrielle. Selon la théorie de la modernisation, la migration a dès lors des effets positifs sur le long terme pour le développement des régions d'origine.

Les critiques ont eu beau jeu de rappeler que, dans bien des cas, l'urbanisation et l'exode rural ne se sont pas accompagnés du développement économique que la théorie de la modernisation annonçait ${ }^{19}$. Ce modèle de la modernisation ne prend pas en considération les contraintes structurelles (comme l'accès inégal aux ressources). Il s'appuie sur l'idée que les personnes ont une capacité égale à prendre des décisions et faillit à reconnaître l'hétérogénéité des sociétés. Les gens ont des aspirations; ils n'agissent pas seulement en fonction d'un calcul coûts-bénéfices. D'ailleurs, plus que les hauts salaires en tant que tels, ce sont les différences de salaires qui attirent les migrants. Contrairement aux théories de l'équilibre, de nombreux migrants se déplacent ainsi de régions peu peuplées vers des régions plus peuplées.

A cette perspective sur les migrations, marquée par une vision bipolaire où les sociétés qui envoient et celles qui reçoivent des migrants sont opposées, s'associe la distinction entre les facteurs push, qui motivent le départ du pays d'origine, et pull, qui attirent le migrant dans le pays d'accueil éventuel. Ce cadre explicatif, qui a connu un énorme succès dans l'étude des migrations sans que

18 D.S. Massey, J. Arango, G. Hugo, A. Kouaouci, A. Pellegrino and J.E. Taylor, «Theories of International Migration: A Review and Appraisal», Population and Development Review, vol. 19, n 3, 1993, pp. 431-466, citation p. 433.

19 Voir par exemple M. Kearney, «From the Invisible Hand to Visible Feet: Anthropological Studies of Migration and Development», Annual Review of Anthropology, vol. 15, 1986, p. 334. 
ses origines théoriques soient clairement identifiables ${ }^{20}$, s'apparente à l'économie néoclassique. En effet, il propose également un modèle d'équilibre fondé sur les décisions individuelles. Ainsi, la croissance démographique en région rurale conduit à une pression sur les ressources. Il s'agit là d'un facteur push: de nombreux membres de la population rurale partent. Symétriquement, les hauts salaires que l'on peut espérer dans les régions industrialisées sont un facteur pull: les migrants s'y rendent dans l'espoir d'améliorer leurs revenus.

Ce modèle peut paraître séduisant par sa simplicité. Il repose toutefois sur une confusion entre différents niveaux d'analyse, en particulier entre les niveaux individuel (ce que les individus perçoivent et prennent en compte dans leurs décisions) et sociétal (les structures du marché de l'emploi par exemple). Comme l'écrit de Haas, "the push-pull model is a static model focusing on external factors that "cause" migration that is unable to analytically situate migration as an integral part of broader transformation processes, and therefore seems of limited analytical use $»^{21}$. En outre, le modèle des facteurs push et pull ne parvient pas à rendre compte de la coexistence au sein d'une même population de mouvements d'émigration et d'immigration. Comment expliquer le retour de nombreux migrants alors que les facteurs push et pull n'ont pas forcément évolué ? Il s'agit d'un modèle plus descriptif qu'analytique, dans lequel les migrations ne sont pas perçues comme un processus; il insiste sur les causes des mouvements, mais semble insuffisant pour rendre compte des modifications induites par les migrations.

\section{La critique des dependentistas}

En réaction à la perspective précédente, des visions plus pessimistes du développement - et pour ce qui nous occupe, de la relation entre migration et développement - se sont dégagées à la fin des années 1960. Ce que l'on a appelé l'école de la dépendance, en particulier, s'est opposé presque point par point à la théorie de la modernisation. Cette école doit beaucoup à l'œuvre de l'historien économique Andre Gunder Frank, qui a parlé du «développement du sous-développement», mais a également connu un grand succès en Amérique latine autour d'un groupe d'intellectuels désignés sous le label de dependentistas (dont le sociologue brésilien Fernando Henrique Cardoso, qui fut président de son pays entre 1995 et 2003) 22. Sur la même lancée, les théoriciens du système monde et les néomarxistes ${ }^{23}$ ont également pris le contre-pied de l'école de la modernisation.

20 Déjà critiquée par Petersen, qui lui reproche de présupposer une tendance universelle à la sédentarité qui n'a de fondement ni historique ni psychologique, la théorie des facteurs push et pull est reprise avec une autre terminologie par Lee, qui cherche à formaliser les facteurs associés à la région d'origine et à la région de destination. Voir W. Petersen, "A General Typology of Migration», American Sociological Review, vol. 23, n 3, 1958, pp. 256-266, et E.S. Lee, «A Theory of Migration», Demography, vol. 3, nº 1, 1966, pp. 47-57.

21 H. de Haas, op. cit., p. 11.

22 A.G. Frank, «The Development of Underdevelopment», Monthly Review, vol. 18, n 4, 1966, pp. 17 31 ; F.H. Cardoso, Les idées à leur place: le concept de développement en Amérique latine, Paris, A.-M. Métailié, 1984.

23 I. Wallerstein, The Modern World-System I: Capitalist Agriculture and the Origins of the European World-Economy in the Sixteenth Century, New York, Academic Press, 1974; The Modern World- 
Ces auteurs partent du constat que le pouvoir politique et économique est inégalement réparti entre les pays développés et sous-développés. Les différentes composantes de la population mondiale n'ont pas un accès identique aux ressources. Bien loin de les résorber, l'expansion du capitalisme renforce ces inégalités au profit du centre et au détriment de la périphérie. Le système mondial se caractérise par des mécanismes d'exploitation, par l'extraction des ressources naturelles et humaines du Sud pour nourrir la croissance du Nord. Le principe du libre-échange est par exemple décrit comme un processus qui tend à favoriser les nantis et à entraver les efforts des populations défavorisées. Nous assistons ainsi à un accroissement de l'asymétrie et non à la mise en place progressive - quoique parfois douloureuse - d'un état d'équilibre. Pour contrer cette évolution inexorable, il est nécessaire de réformer le régime d'appropriation des surplus et d'accumulation qui prévaut dans les pays occidentaux. Il faut donc faire cesser l'utilisation systématique des ressources naturelles et humaines de la périphérie par le centre en octroyant aux Etats un rôle régulateur accru et en encourageant l'industrialisation des pays du tiers-monde pour leur permettre de se développer à leur tour.

Dans un tel cadre conceptuel, les migrations internationales ne sont qu'une des faces des rapports globaux de domination. Elles ont des effets négatifs sur le développement des pays du Sud; elles participent à l'élargissement de l'écart entre le centre et la périphérie, mais aussi à la rupture des liens de solidarité dans les sociétés d'origine des migrants. Contrairement à ce que les théories exposées précédemment préconisent, les migrants ne circulent pas en direction opposée aux capitaux, mais se rendent où se trouve le capital. Les individus ne sont pas libres de leurs choix, mais sont contraints par des forces structurelles sur lesquelles l'analyse doit se concentrer. Les flux migratoires participent dès lors à l'aggravation des problèmes de sous-développement et de la situation de dépendance des pays du tiers-monde; ils représentent pour ces derniers une fuite devant la misère, une perte de capital humain et donc le départ des forces vives d'une sociétée ${ }^{4}$.

On a pu reprocher à cette approche d'être déterministe et trop globalisante pour fournir de bonnes pistes de recherche pour des terrains spécifiques. Elle ne prend pas en compte les stratégies de résistance ou de réappropriation de la périphérie. Elle repose en effet sur une vision simplificatrice de la division internationale du travail et conçoit le monde comme ayant un centre unique, alors qu'aujourd'hui il est difficile de ne pas reconnaître son caractère multicentrique. En outre, elle réduit les être humains au statut de victimes de forces structurelles qui les dépassent et nie leur capacité d'action. Elle néglige parallèlement les facteurs culturels et exagère l'importance des questions économiques aux dépens des relations

System II: Mercantilism and the Consolidation of the European World-Economy, 1600-1750, New York, Academic Press, 1980; The Modern World-System III: The Second Era of Great Expansion of the Capitalist World-Economy, 1730-1840s, New York, Academic Press, 1989.

24 Cette vision négative des migrations Sud-Nord est toujours largement répandue parmi les intellectuels africains. Beaucoup considèrent que les départs massifs vers l'Europe sont le résultat des rapports de domination et de la recolonisation menée sous l'égide de la mondialisation libérale, qui conduit à la paupérisation et à l'exclusion de larges franges de la population mondiale. Ils militent pour que les Africains se réapproprient leur destin et prennent en main le développement de leur continent. Voir par exemple Aminata Traoré, L'Afrique humiliée, Paris, Fayard, 2008, ou Jean-Emmanuel Pondi (dir.), Immigration et diaspora: un regard africain, Paris; Yaoundé, Maisonneuve et Larose, 2007. 
politiques $^{25}$. Finalement, les dependentistas ont dénoncé les mécanismes causant le sous-développement et ont milité pour la suppression des obstacles structuraux et historiques qui maintenaient le tiers-monde dans un état de sujétion, mais ils n'ont pas réellement su remettre en question la conception dominante du développement comme la promesse d'un bonheur généralisé ${ }^{26}$.

\section{Une approche pluraliste}

Cette brève présentation permet de montrer qu'il est indispensable d'aller audelà de l'opposition entre des visions monoparadigmatiques, que ce soient celle de la modernisation ou celle de la dépendance. La relation entre migration et développement est plus complexe et moins linéaire que ne le laissent entrevoir ces positions classiques ou l'optimisme institutionnel qui domine aujourd'hui. Aucune réponse simple ne peut être donnée; il n'y a pas de lien automatique, mécanique ou déterminé entre les deux types de phénomènes, ni d'ailleurs entre migration et pauvreté. Une approche pluraliste et flexible est nécessaire; elle doit permettre de prendre en considération la variation des cas dans le temps et dans l'espace mais aussi d'appréhender de façon générale la relation entre migration et développement ${ }^{27}$.

La valorisation contemporaine du rôle des migrants sur la scène politique tend en effet à séparer l'étude des causes et des conséquences des migrations en nourrissant l'idée que résoudre les problèmes dans les pays d'origine permettrait d'endiguer les flux migratoires. Cela conduit à négliger le fait que les processus migratoires ont leurs dynamiques propres, qu'ils peuvent se reproduire de façon relativement autonome, et qu'ils ne peuvent pas être réduits à de simples facteurs économiques ni à l'agrégation de choix individuels. En outre, les migrations répondent à des raisons structurales autant des pays de destination (marché de l'emploi, déséquilibres démographiques, etc.) que des pays de départ (pauvreté, sous-développement, etc.).

Une raison supplémentaire de complexifier notre conception de la relation entre migration et développement découle de la transition démographique que traversent les populations qui bénéficient d'une amélioration de leurs conditions de vie. A un stade initial, la situation est caractérisée par de forts taux de natalité et de mortalité. Par contre, le taux de croissance de la population est faible. Les progrès sanitaires, une alimentation améliorée ou la scolarisation vont provoquer une baisse rapide du taux de mortalité, alors que la natalité ne va pas, dans un premier temps, varier de façon aussi importante. La population va connaître un accroissement rapide provoquant un excédent démographique qui n'est pas toujours comblé par une meilleure productivité agricole, par les emplois créés

25 M. Kearney, op. cit., pp. 331-361.

26 G. Rist, op. cit., pp. 194-195.

27 Voir les travaux de H. de Haas, un auteur déjà mentionné, pour une présentation critique de la litérature: Engaging Diasporas: How Governments and Development Agencies Can Support Involvement in the Development of Origin Countries, Oxford, International Migration Institute, University of Oxford, 2006; Turning the Tide? Why «Development instead of Migration» Policies are Bound to Fail, Oxford, International Migration Institute, University of Oxford, 2006; Remittances, Migration and Social Development: A Conceptual Review of the Literature, Geneva, United Nations Research Institute for Social Development, 2007; Migration and Development, op. cit. 
dans le secteur secondaire ou par le renforcement du tertiaire. C'est la situation de déséquilibre dont l'Europe a fait l'expérience au XIX ${ }^{\mathrm{e}}$ siècle et qu'elle a gérée par le départ de plusieurs dizaines de millions de personnes vers le NouveauMonde. L'émigration a clairement fonctionné ici comme un exutoire de l'augmentation de la population. Dans une phase ultérieure, la mortalité continue à baisser, mais plus lentement, alors que la natalité commence à son tour à décroître de façon sensible. Les deux courbes vont finalement se stabiliser à un bas niveau. Le taux de croissance de la population va faiblir à nouveau et parfois atteindre des valeurs négatives, situation que l'Occident connaît aujourd'hui ${ }^{28}$. Les pays du Sud, par contre, sont en pleine transition démographique mais n'ont pas de débouchés migratoires comparables à ceux que l'Europe s'était créés entre les guerres napoléoniennes et le premier conflit mondial.

La pression démographique liée à cette période de transition que connaissent les régions dans une phase initiale de croissance économique n'est pas la seule raison qui explique un flux d'émigration accru. En effet, un décollage économique va augmenter les moyens que les gens ont à disposition pour migrer. $\mathrm{Ce}$ ne serait ainsi qu'à un niveau supérieur de développement économique que l'émigration diminuerait. C'est ce qui a été nommé le migration hump dans la littérature de langue anglaise. En fait, plus qu'une diminution tranchée des flux migratoires, les observations effectuées montrent que les modalités de déplacement et les parcours se diversifient lorsque les migrants et les populations dont ils sont issus consolident leurs ressources financières.

\section{Schéma 1: Les effets du développement sur les flux migratoires - le migration hump}
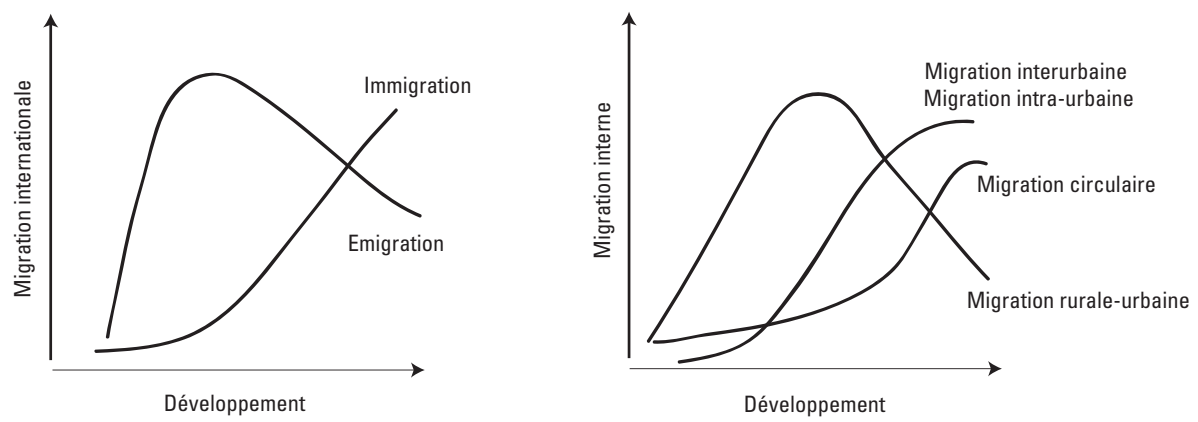

Source: Hein de Haas, Migration and Development: A Theoretical Perspective, Oxford, University of Oxford, 2007, p. 18.

Il n'y a donc pas de relation inversement proportionnelle entre migration et développement, ni de façon plus spécifique de rapport univoque entre migration et pauvreté. La relation dépend du contexte et peut grandement varier d'une région à l'autre et au cours du temps. En outre, elle est réversible, une situation de baisse de développement ou de conflit peut transformer des pays d'immigration en pays d'émigration, comme dans le cas de la Côte d'Ivoire ou de la République démocratique du Congo en Afrique. Le lien causal entre les deux catégories de

28 Dans un tel contexte, notons que $56 \%$ de l'accroissement de la population dans les pays développés résulte des migrations internationales entre 1990 et 2000 ; ce pourcentage se monte même à $89 \%$ pour 1'Europe et à $75 \%$ pour l'Amérique du Nord entre 1995 et 2000. Voir Migration in an Interconnected World, op. cit., pp. 84-85. 
phénomènes n'est donc pas déterminé. En fait, le type de changements sociaux et économiques que l'on nomme développement a tendance dans un premier temps à augmenter la capacité des gens à migrer. Sur le long terme, on assiste à une diversification des modèles migratoires ${ }^{29}$.

Ces quelques considérations nous conduisent à prendre le contre-pied de certaines idées reçues, comme celle selon laquelle la pauvreté, mesurée selon des critères standardisés et généraux, est la principale cause des migrations SudNord. Plus décisifs sont les différentiels, autrement dit les niveaux relatifs de pauvreté. D'autres facteurs, comme le taux de scolarisation ou l'espérance de vie, jouent également un rôle important. Les effets du développement sur les migrations sont donc contrastés. Une perspective moins idéologique, sensible aux différences régionales, permet de dépasser les débats piégés entre les optimistes et les pessimistes. Pour que cet effort de distanciation par rapport aux discours qui dominent la scène politique et institutionnelle soit possible, il est nécessaire de disposer d'un cadre théorique et analytique suffisamment flexible pour rendre compte des variations et de s'appuyer sur des recherches empiriques fouillées qui permettront de dresser l'inventaire du champ des possibles. Il s'agit de prendre en compte les initiatives des migrants et des non-migrants (leur capacité d'action pour faire évoluer le cadre structurel et institutionnel dans lequel ils évoluent), mais aussi des contraintes structurelles auxquelles ils sont confrontés (marché, rapports de pouvoir globaux et locaux, etc.).

Un tel programme de recherche peut être mis en place en analysant les systèmes migratoires dans leur dimension transversale, et non en se penchant alternativement sur les pays de départ ou de destination des migrants. Certains lieux sont en effet reliés par des systèmes d'échanges qui nous permettent d'identifier des ensembles migratoires particuliers, quoique poreux :

- le système américain, qui unit les Etats Unis et le Canada à l'Amérique latine par le Mexique (pays charnière, qui est à la fois une zone d'origine, de transit et de destination);

๑ le système euroméditerranéen, qui comporte deux grandes routes migratoires : celle qui relie l'Afrique subsaharienne à l'Europe par le Maroc et la Tunisie; celle qui, du Moyen-Orient, passe par la Turquie et les Balkans ${ }^{30}$;

- le système centré sur les pays du golfe Persique, où affluent de nombreux migrants originaires du reste du Moyen-Orient, du sous-continent indien et de l'Asie du Sud-Est;

ـ le système qui, de l'ensemble de l'Asie, mène vers l'Australie mais aussi vers le Japon par le Sud-Est asiatique.

Si ces ensembles forment système, il est fécond d'étudier ce qui se passe dans les pays de départ ou de transit en faisant référence à ce qui se passe à destination, et vice versa. Toutes les étapes des parcours migratoires sont en effet connectées. Parallèlement, le fait d'appréhender conjointement les causes et les conséquences de ces flux et de relier migrants et non-migrants, régions d'origine et d'accueil permet de mettre en lumière les contextes globaux de migration et de développement.

29 H. de Haas, Migration and Development, op. cit., p. 17.

30 Voir l'ouvrage récent d'A. Tarrius, La remontée des Sud: Afghans et Marocains en Europe méridionale, La Tour-d'Aigues, Editions de l'Aube, 2007. 


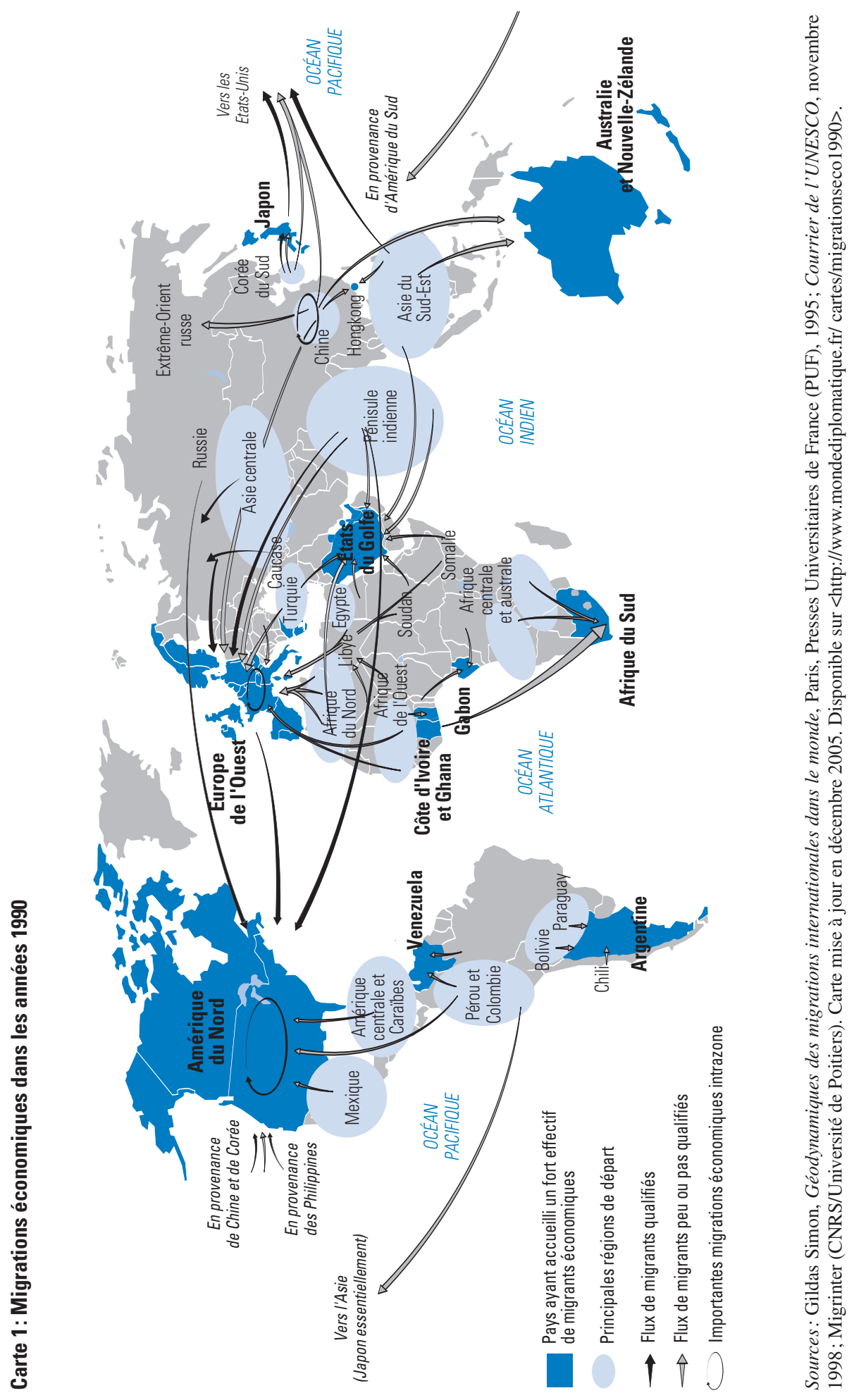


Indépendamment des débats sur le développement, l'étude des migrations a connu depuis le milieu des années 1980 un essor spectaculaire, en lien avec la période de doutes et de déconstruction que traversent les sciences sociales à cette époque. De façon croissante, la mobilité des personnes n'est plus considérée comme le déplacement entre un point $\mathrm{A}$ et un point $\mathrm{B}-$ l'intégration des migrants pouvant être plus ou moins réussie au terme de ce processus unidirectionnel -, mais comme un phénomène complexe caractérisé par une circulation continue ${ }^{31}$. Certains auteurs nomment «transmigrants» les personnes qui développent et maintiennent des relations multiples - qu'elles soient familiales, économiques, sociales, religieuses ou politiques - au-delà des frontières étatiques, culturelles et géographiques. Autrement dit, il s'agit de personnes qui tissent des liens multiples entre de nombreux endroits et dont la vie sociale s'organise en des réseaux qui s'étendent simultanément sur plusieurs Etats-nations ${ }^{32}$.

Dans cette perspective que l'on désigne aujourd'hui par le terme de «transnationalisme», les groupes socioculturels ne doivent plus être conçus comme des entités séparées les unes des autres et délimitées territorialement. En allant audelà de l'opposition tranchée entre société d'origine et société de destination, cette approche permet aussi de dépasser les catégories formelles de migrations permanentes et temporaires, forcées et volontaires, et également de ne plus considérer l'intégration dans le pays de destination ou le retour dans le pays de départ comme la fin du cycle migratoire. Toute une nouvelle génération de chercheurs utilise ainsi le concept de réseau transnational pour étudier les migrations forcées ${ }^{33}$. Sans nier la spécificité des réfugiés en termes légaux ni les périls aux-

31 Pour une présentation et une appréciation générales de ce tournant, voir: S. Vertovec, «Conceiving and Researching Transnationalism», Ethnic and Racial Studies, vol. 22, n 2, 1999, pp. 447-462. Parmi les nombreux ouvrages récents qui abordent ces questions, mentionnons: N. Al-Ali and Kh. Koser (eds.), New Approaches to Migration? Transnational Communities and the Transformation of Home, London; New York, Routledge, 2002; Th. Faist, The Volume and Dynamics of International Migration and Transnational Social Spaces, Oxford, Oxford University Press, 2000.

Une telle perspective remet en question le modèle migratoire classique de l'intégration, qui inspire la plupart des politiques nationales, mais aussi une certaine conception de la citoyenneté et de l'Etatnation. Voir Th. Faist and P. Kivisto (eds.), Dual Citizenship in a Global Perspective: From Unitary to Multiple Citizenship, New York, Palgrave Macmillan, 2007.

32 N. Glick Schiller, L. Basch and C. Blanc-Szanton (eds.), Towards a Transnational Perspective on Migration: Race, Class, Ethnicity, and Nationalism Reconsidered, New York, The New York Academy of Sciences, 1992; et, des mêmes auteurs, «From Immigrant to Transmigrant: Theorizing Transnational Migration», Anthropological Quarterly, vol. 68, n 1, 1995, pp. 48-63.

33 Dans cette abondante littérature, citons: N. Al-Ali, R. Black and Kh. Koser, «Refugees and Transnationalism: The Experience of Bosnians and Eritreans in Europe», Journal of Ethnic and Migration Studies, vol. 27, $\mathrm{n}^{\circ}$ 4, 2001, pp. 615-634; G. Chatelard, Iraqi Forced Migrants in Jordan: Conditions, Religious Networks, and the Smuggling Process, Working Papers, $\mathrm{n}^{\circ}$ 49, Florence, Robert Schuman Center for Advanced Studies, European University Institute, 2002; C. Horst, Transnational Nomads: How Somalis Cope with Refugee Life in the Dadaab Camps of Kenya, New York; Oxford, Berghahn Books, 2006; E. Marx, «The Social World of Refugees : A Conceptual Framework», Journal of Refugee Studies, vol. 3, no 3, 1990, pp. 189-203; A. Monsutti, Guerres et migrations : réseaux sociaux et stratégies économiques des Hazaras d'Afghanistan, Neuchâtel, Institut d'ethnologie; Paris, Maison des sciences de l'homme, 2004, et «Afghan Migratory Strategies and the Three Solutions to the Refugee Problem», Refugee Survey Quarterly, vol. 27, n 1, 2008, pp. 58-73; S. Shami, «Transnationalism and Refugee Studies: Rethinking Forced Migration and Identity in the Middle East», Journal of Refugee Studies, vol. 9, nº 1, 1996, pp. 3-26. 
quels ils font face, ces auteurs se sont inspirés des considérations théoriques et méthodologiques développées en premier lieu dans le champ des migrations volontaires. Cette approche se justifie par plusieurs constats. Tout d'abord, les personnes reconnues comme réfugiées ne sont pas les pures victimes d'un sort qui les dépasse, mais des acteurs qui s'efforcent de réagir à des conditions difficiles en s'appuyant sur les ressources sociales et culturelles qui restent à leur disposition. Ensuite, les stratégies qu'elles développent sont souvent similaires à celles des personnes considérées comme des travailleurs migrants. Finalement, les frontières entre les divers statuts (personnes déplacées de l'intérieur, réfugiés, travailleurs migrants) sont perméables; en s'adaptant à un contexte sans cesse changeant et en jouant avec les étiquettes, les mêmes personnes peuvent appartenir à diverses catégories, à la fois ou successivement.

Dans une perspective transnationale, tous les lieux concernés seront étudiés comme un système, un ensemble. En effet, la dispersion des groupes familiaux est souvent le résultat d'une stratégie pour diversifier les ressources et diluer les risques; elle ne s'accompagne dès lors pas forcément d'un affaiblissement des relations. Comme nous l'avons mentionné précédemment, cela correspond aux principes de la new economics of labor migration (NELM) ${ }^{34}$. Alors que l'économie néoclassique est centrée sur l'individu et ses choix rationnels, la NELM prend en considération le ménage - dans sa variété socioculturelle - comme unité décisionnelle de base. Plutôt que de maximiser son intérêt, il s'agit ici de diminuer les risques et d'avoir accès à une plus grande variété de ressources. Dans une telle perspective, les remittances occupent une place centrale, car ils permettent de diversifier et d'augmenter les revenus du ménage, de contourner les difficultés et les obstacles économiques locaux (en particulier un accès difficile au crédit). Par la dispersion planifiée des membres d'une unité domestique, la migration apparaît ici comme une stratégie de diversification du capital social et économique; autrement dit, comme une assurance contre la détérioration toujours possible des conditions de vie sur le lieu d'origine. Elle constitue ainsi une véritable livelihood strategy $y^{35}$.

Mais en quoi ces considérations sur le transnationalisme, la nouvelle économie des migrations et les stratégies de subsistance éclairent-elles la problématique de la relation entre migration et développement? Elles conduisent en particulier à accepter l'idée que l'insertion économique et sociale réussie d'un migrant dans une société d'accueil n'aboutit pas forcément à une interruption des relations avec sa société d'origine. Il peut avoir un engagement complémentaire et concomitant dans deux sociétés ou plus. Un migrant intégré peut ainsi rester un acteur du développement dans sa société d'origine, mais également rester actif dans la vie politique. Contrairement à la vision proposée par les partisans de la théorie de la modernisation, il ne faut pas nécessairement rentrer dans sa société d'origine pour être un acteur du développement. Contrairement à

34 O. Stark and D.E. Bloom, «The New Economics of Labor Migration», American Economic Review, vol. 75, nº 2, 1985, pp. 173-178; voir également D.S. Massey et al., op. cit., pp. 440-442.

35 Dans la littérature développementaliste de langue anglaise, on entend par livelihood l'ensemble des moyens (ressources matérielles et sociales) qui permettent de subvenir à ses moyens. Cela inclut notamment l'emploi, les institutions, les relations interpersonnelles et interfamiliales. On parle souvent aujourd'hui - parfois avec un certain manque de précision - de capital naturel, social, humain, physique, économique ou financier. Par livelihood strategy, on réfère au choix planifié pour combiner différentes ressources et activités. 
l'école dépendantiste, le fait de migrer n'est pas le signe d'une absence de capacité d'action. Cette perspective est également en porte-à-faux avec les chercheurs qui prédisent que les migrants vont graduellement couper leurs liens avec le milieu d'origine. L'approche transnationale montre au contraire que les expériences et les contacts des premiers migrants orientent les itinéraires des migrants successifs. Ces derniers bénéficient d'informations sur les possibilités d'emploi, sur les emplacements où ils peuvent se rendre pour espérer être aidés, pour trouver un logement, des papiers d'identité ou un permis de séjour. On prend en compte ici les facteurs qui tendent à perpétuer la migration, comme les stratégies de relais migratoire: une rotation planifiée des membres de la famille se met en place, chacun allant à tour de rôle à l'étranger à la recherche d'un emploi rémunéré dont tout le monde profite; le retour d'un membre de l'unité domestique est ainsi souvent concomitant avec le départ d'un autre ${ }^{36}$. Bref, on reconnaît ici aux réseaux sociaux un grand rôle. Ils facilitent le flux d'informations entre les migrants et les non-migrants et permettent de rendre compte des envois massifs que les migrant effectuent vers leurs lieux d'origine.

\section{Transferts de fonds et développement}

Les discussions concernant les effets des remittances dans la lutte contre la pauvreté et plus généralement leur apport pour l'économie et la société des pays d'origine cristallisent en grande partie les débats sur la relation entre migration et développement. Beaucoup mieux que la théorie de la modernisation, pour laquelle les migrants jouent essentiellement un rôle moteur dans le développement de leurs régions d'origine après y être retournés, ou que la théorie de la dépendance, qui ne voit dans les migrations internationales que le signe de la décomposition des économies et des sociétés du Sud, l'approche transnationale et la nouvelle économie des migrations mettent en lumière les liens sociaux qui sous-tendent les très importants transferts de fonds effectués par les migrants.

Comme nous l'avons vu (tableau 1), les montants impliqués sont extrêmement importants; ils sont en particulier nettement plus élevés que l'APD. Selon de nombreux chercheurs, dont le discours est relayé par les institutions de développement, les remittances constituent dès lors une stratégie de survie efficace et un moyen incontournable pour lutter contre la pauvreté. Ils sont aussi sensibles à la cohésion sociale que cette vaste circulation de biens reproduit ${ }^{37}$.

36 H. de Haas, Migration and Development, op. cit., pp. 19, 22 ; D.S. Massey et al., op. cit., pp. 460-462. Pour la stratégie de relais migratoire des Afghans qui se rendent en Iran, voir A. Monsutti, Guerres et migrations, op. cit., pp. 207, 209 et 225 par exemple.

37 Il existe une volumineuse littérature portant sur les transferts de fonds des migrants, la réduction de la pauvreté et le développement, dont de nombreux rapports de la Banque mondiale, de l'Organisation internationale pour les migrations et de diverses agences nationales de coopération (comme le DFID britannique). Par exemple: Committee on Payment and Settlement Systems and World Bank, General Principles for International Remittance Services, Basel, Bank for International Settlements; Washington, DC, World Bank, March 2006. Mentionnons également le numéro spécial intitulé The MigrationDevelopment Nexus de International Migration, vol. 40, $\mathrm{n}^{\circ}$ 5, 2002; ainsi qu'un article qui fait le point des aspects économiques: R.H. Adams and J. Page, «Do International Migration and Remittances Reduce Poverty in Developing Countries?», World Development, vol. 22, n 10, 2005, pp. 1645-1669. Voir également les divers textes de R. Ballard disponibles sur le site du Centre for Applied South Asian Studies, University of Manchester, <http://www.art.man.ac.uk/CASAS/pages/papers.htm>. 
Ne cédons toutefois pas à l'angélisme. Pour d'autres auteurs, les versements des migrants ne sont pas investis dans des activités productives, mais nourrissent des dépenses ostentatoires, l'acquisition de biens de consommation ou de prestige, de biens immobiliers. Ils ne contribueraient donc guère au développement économique des pays d'origine des migrants Loin de dynamiser la production et de participer à la création d'emplois, l'argent des migrants contribuerait à l'inflation et marquerait la dépendance face au monde extérieur. Comme ce ne sont pas les plus pauvres qui migrent et qui bénéficient des transferts de fonds, ces derniers contribueraient à creuser les inégalités. Certains systèmes informels de transfert seraient même des outils de blanchiment d'argent sale et permettraient aux réseaux terroristes de faire circuler discrètement leurs avoirs ${ }^{38}$. Dans cette perspective, l'une des seules conséquences positives de l'argent envoyé par les migrants serait de faciliter la scolarisation des enfants ${ }^{39}$.

Le rôle des transferts de fonds par les migrants dans les situations de conflit a également été débattu, sans toutefois recevoir une attention comparable ${ }^{40}$. L'argent des migrants peut certes contribuer à relancer la vie économique en situation de conflit ou de paix encore fragile ${ }^{41}$. Toutefois, il risque toujours d'être capté par des factions combattantes et de nourrir ainsi les luttes intestines ${ }^{42}$. Dans des situations où l'Etat est faible, certains leaders ou groupes politiques parviennent à accaparer les ressources et à contrôler les canaux de distribution des marchandises et des services pour ensuite les redistribuer et accroître leur influence politique.

Les arguments des uns et des autres sont résumés dans le tableau 2 (page suivante).

Cette énumération suffit à démontrer que si les transferts financiers et sociaux des migrants offrent un réel potentiel pour le développement des régions d'origine, ils présentent également des dangers. Il serait vain de prendre une position définitive face à cette discordance d'arguments et à la diversité des cas.

38 Ce reproche est souvent fait au système hawâla, qui s'est dans le même temps avéré être une très efficace stratégie de survie pour les Afghans (voir ma contribution sur ce thème dans ce volume).

39 Voir par exemple M. Kearney, op. cit., pp. 346-347; ou M.-A. Pérouse de Montclos, «Réseaux financiers, diasporas et hawilad: le rôle clé de la péninsule Arabique entre l'Occident et la Somalie», in E. Grégoire et J. Schmitz (dir.), Afrique noire et monde arabe: continuités et ruptures, Paris, Aube; IRD, 2000, pp. 114-115.

40 Voir P. Collier, Economic Causes of Civil Conflict and Their Implications for Policy, Washington, DC, World Bank, 2000, et ses critiques, R. Marchal et Ch. Messiant, «De l'avidité des rebelles: l'analyse économique de la guerre civile selon Paul Collier», Critique internationale, vol. 16, juillet 2002, pp. 59-69. Ces auteurs s'intéressent toutefois plus au rôle des groupes armés qu'à celui des diasporas.

41 Voir un rapport publié dans le cadre du Security-Development Nexus Programme de l'International Peace Academy: P. Weiss Fagen and M.N. Bump, Remittances in Conflict and Crises: How Remittances Sustain Livelihoods in War, Crises, and Transitions to Peace, Washington, DC, International Peace Academy, February 2006.

42 Les Tigres de libération de l'Eelam Tamoul (plus connus sous l'acronyme anglais de LTTE), le Parti des travailleurs du Kurdistan (PKK) ou l'Armée de libération du Kosovo (UÇK) ont par exemple été soupçonnés de se financer en partie avec l'argent des migrants. 
Tableau 2: Points positifs et négatifs des remittances pour le développement

Points positifs

- Stratégie efficace de survie.

- Diversification des sources de revenu; la migration comme une forme d'assurance sociale.

- Réduction de la pauvreté dans le pays d'origine.

- Augmentation du pouvoir d'achat; effets indirects multiplicateurs sur l'économie.

- Investissements potentiellement productifs (correspondant à environ 20\% des transferts).

- Création de petites entreprises.

- Compensation de la perte de revenu due au conflit.

- Développement communautaire par des associations de migrants.

- Promotion de l'éducation (parmi les membres des familles des migrants qui sont restés dans le pays d'origine); transfert de connaissances et retour de migrants rapportant les compétences apprises à étranger (brain gain); impact positif sur la santé, sensibilisation aux soins. .

- Promotion de la paix, des droits de l'homme, de la démocratie (social remittances).

- Dimension sociale: cohésion des groupes de solidarité malgré la dispersion; établissement de réseaux transnationaux.

Points négatifs

- Départ de la force vive d'une société, de la main-d'œuvre (particulièrement lourd dans les économies fondées sur l'agriculture).

- Création d'une culture de la dépendance.

- Investissement dans l'immobilier et dans les biens de consommation; augmentation du coût de la vie (en particulier les loyers).

- Dimension privée des transferts; faibles retombées communautaires.

- Accroissement des inégalités: les ménages les plus pauvres n'ont pas les moyens d’envoyer l'un de leurs membres à l'étranger; élargissement du fossé entre les nantis et les miséreux.

- Exode des personnes formées (brain drain) ou non-insertion des migrants qui retournent avec de nouvelles compétences acquises à l'étranger (brain waste).

- Contrairement à la théorie de la subsidiarité de la Banque mondiale, les migrants ayant un niveau élevé de formation n'envoient d'ailleurs pas beaucoup d'argent dans leurs pays d'origine.

- Alimentation de la corruption.

- Financement de groupes armés; outil des réseaux terroristes internationaux.

- Blanchiment d'argent lié à des activités criminelles.

- Risque d'encourager les réseaux de traite humaine (human trafficking) ou d'être en lien avec eux.

\section{Conclusions et perspectives}

Comme nous l'avons vu, la relation entre migration et développement est complexe et ne se laisse pas appréhender par des visions tranchées, qu'elles soient optimistes ou pessimistes. Il faut plutôt militer pour une approche pluraliste et flexible, apte à prendre en considération la variation des situations dans le temps et dans l'espace. L'optimisme institutionnel qui prévaut aujourd'hui méconnaît les débats théoriques qui ont animé les sciences sociales depuis les années 1950. En outre, il en reste bien souvent au niveau des énoncés d'intention et n'inspire que rarement les politiques de façon concrète. Cette myopie historique et ce décalage entre discours et pratiques laissent perplexe.

L'approche transnationale souligne que les migrants tissent des liens multiples au cours de leurs parcours et qu'ils sont bien souvent acteurs à part entière de plusieurs sociétés à la fois. Afin de saisir la complexité des phénomènes, il est nécessaire de décloisonner les catégories de migrants et de reconnaître la nonlinéarité des itinéraires: les réfugiés peuvent se transformer en entrepreneurs et envoyer de l'argent dans leur lieu d'origine; parallèlement, des étudiants peuvent faire quelques économies pendant leurs séjours à l'étranger et envoyer des 
remittances à leurs familles. Il est indéniable que les migrants et les réfugiés peuvent dès lors jouer un rôle dans le développement économique de leurs pays de départ. Mais la relation entre migration et développement n'est pas inversement proportionnelle. Les recherches empiriques existantes ont montré que la variété des cas ne se laissait pas réduire facilement; elles n'ont pas établi de rapport univoque et absolu entre transferts de fonds et réduction de la pauvreté ou entre croissance économique et diminution des flux migratoires. D'ailleurs, les écarts de richesse semblent plus directement décisifs que la pauvreté en tant quel telle. La migration n'est pas forcément une fuite désepérée devant la misère ou la violence; elle découle souvent d'une volonté de diversification géographique, sociale, économique et politique pour dépasser les contraintes locales. La dispersion spatiale des membres d'un groupe domestique constitue ainsi une assurance contre une évolution négative du cadre de vie dans le lieu d'origine.

Dans une telle perspective, toute la palette de cas possibles, allant de la migration pleinement volontaire à l'exode de réfugiés fuyant des bombardements, est reconnue. La mobilité est souvent une option assumée qui s'insère dans le parcours de vie de gens et contribue à redéfinir les rapports entre générations comme entre genres. Elle peut permettre par exemple à des jeunes hommes de construire leur masculinité et d'affirmer leur statut d'adulte ${ }^{43}$. De très nombreuses femmes migrent quant à elles de façon autonome, alors que celles dont le mari est parti peuvent acquérir de nouvelles responsabilités, que ce soit dans la gestion du ménage, les travaux agricoles ou plus généralement les processus de décision qui concernent la famille. La restructuration économique globale aboutit à une nouvelle division internationale du travail. Qu'elles soient migrantes ou non-migrantes, les femmes - et les jeunes - ont ainsi la possibilité de contester les anciennes hiérarchies et de conquérir de nouveaux espaces de liberté ${ }^{44}$.

La migration, hier comme aujourd'hui, est présentée ici comme un élément constitutif des sociétés humaines. L'intérêt contemporain pour les remittances est à ce titre encourageant, car il s'agit par là de reconnaître les apports de la mobilité. Le danger d'une dérive et d'une instrumentalisation politique existe cependant. Les transferts de fonds des migrants permettent certes de minimiser les risques et d'augmenter les revenus des ménages qui en bénéficient. Mais cela ne signifie pas qu'ils contribuent de façon déterminante et globale à lutter contre la pauvreté ni à accroître le PNB des pays du Sud. Ils ne peuvent résoudre les problèmes structuraux du sous-développement auxquels l'APD est censée s'attaquer. Il faut donc rester circonspect et se méfier de l'optimisme ambiant pour préférer une approche pluraliste de la relation entre migration et développement. Aucune généralisation hâtive ne peut être faite, car les apports des migrants à leurs sociétés d'origine varient de cas en cas.

Célébrer sans ambages les migrations internationales et les transferts de fonds comme la nouvelle solution du sous-développement des pays du Sud dénote pour le moins d'une absence suspecte de perspective historique et théorique.

43 Voir par exemple F. Osella and C. Osella, «Migration, Money and Masculinity in Kerala », Journal of the Royal Anthropological Institute, vol. 6, 2000, pp. 117-133; A. Monsutti, «Migration as a Rite of Passage: Young Afghans Building Masculinity and Adulthood in Iran », Iranian Studies, vol. 40, $\mathrm{n}^{\circ} 2$, 2007, pp. 167-185.

44 Il existe une vaste littérature qui aborde la redéfinition des rapports de genre par la migration. Rappelons par exemple l'ouvrage déjà cité de B. Ehrenreich et A.R. Hochschild. 
Cela comporte également une contradiction interne, puisque la migration devient le principe de sa négation: d'une part, les migrants sont présentés comme les «héros cachés du développement international»; d'autre part, le développement est censé entraîner une diminution des migrations globales. Sans vouloir finir par une provocation, laissons-nous inspirer par un auteur comme Thomas Faist, qui va jusqu'à se demander si les discours qui insistent sur les liens entre migration et développement ne servent pas, en dernier ressort, à légitimer la volonté politique accrue de contrôler et limiter les flux migratoires, au Nord comme au Sud ${ }^{45}$. Question impertinente et dérangeante certes, mais qui mérite d'être posée!

\section{Bibliographie sélective}

Adams, R.H. and J. Page, «Do International Migration and Remittances Reduce Poverty in Developing Countries?», World Development, vol. 22, n 10, 2005, pp. 1645-1669.

Ballard, R., «Migration, Remittances, Economic Growth and Poverty Reduction: Reflections on Some South Asian Developments», in T. Siddiqui (ed.), Migration and Development: Pro-Poor Policy Choices, Dhaka, The University Press Ltd, 2005, pp. 333-358.

Faist, Th., «Migrants as Transnational Development Agents: An Inquiry into the Newest Round of the Migration-Development Nexus », Population, Space and Place, vol. 14, 2008, pp. 21-42.

Global Commission on International Migration, Migration in an Interconnected World: New Directions for Action, Geneva, Global Commission on International Migration, October 2005.

Gosh, B., Migrants' Remittances and Development: Myth, Rhetoric and Realities, Geneva, International Organization for Migration; The Hague, The Hague Process on Refugees and Migration, 2006.

Haas, H. de, Engaging Diasporas: How Governments and Development Agencies Can Support Involvement in the Development of Origin Countries, Oxford, International Migration Institute, University of Oxford, 2006.

Haas, H. de, Migration and Development: A Theoretical Perspective, Oxford, International Migration Institute, University of Oxford, 2007.

Haas, H. de, Turning the Tide? Why «Development Instead of Migration» Policies are Bound to Fail, Oxford, International Migration Institute, University of Oxford, 2006.

Kapur, D., Remittances: The New Development Mantra?, G-24 Discussion Paper Series, no 29, New York; Geneva, United Nations Conference on Trade and Development, 2004.

Kearney, M., «From the Invisible Hand to Visible Feet: Anthropological Studies of Migration and Development», Annual Review of Anthropology, vol. 15, 1986, pp. 331-361.

Massey, D.S., J. Arango, G. Hugo, A. Kouaouci, A. Pellegrino and J.E. Taylor, «Theories of International Migration: A Review and Appraisal», Population and Development Review, vol. 19, $\mathrm{n}^{\circ}$ 3, 1993, pp. 431-466,

Nyberg-Sørensen, N., N. Van Hear and P. Engberg-Pedersen, «The Migration-Development Nexus : Evidence and Policy Options », International Migration, vol. 40, $\mathrm{n}^{\circ}$ 5, 2002, pp. 49-73.

Nyberg-Sørensen, N., N. Van Hear and P. Engberg-Pedersen, «The Migration-Development Nexus: Evidence and Policy Options - State-of-the-art Overview », International Migration, vol. 40, $\mathrm{n}^{\circ}$ 5, 2002, pp. 3-47.

Pessar, P. and S.J. Mahler, «Transnational Migration: Bringing Gender In», International Migration Review, vol. 37, $\mathrm{n}^{\circ} 3,2003$, pp. 812-846.

Ratha, D., «Workers' Remittances : An Important and Stable Source of External Development Finance», Global Development Finance 2003, Washington, DC, World Bank, 2003, pp. 157-175

Vertovec, S., «Conceiving and Researching Transnationalism», Ethnic and Racial Studies, vol. 22, $\mathrm{n}^{\circ} 2$, 1999, pp. 447-462.

Weiss Fagen, P. and M.N. Bump, Remittances in Conflict and Crises: How Remittances Sustain Livelihoods in War, Crises, and Transitions to Peace, Washington, DC, International Peace Academy, February 2006.

45 Th. Faist, «Migrants as Transnational Development Agents», op. cit., p. 38. 